\title{
Article
}

\section{Microwave-assisted rapid synthesis of \#-cyclodextrin metal- organic frameworks for size control and efficient drug loading}

Botao Liu, Yaping He, Liping Han, Vikramjeet Singh, Xiaonan Xu, Tao

Guo, Fanyue Meng, Xu Xu, Peter York, Zhaoxin Liu, and Jiwen Zhang

Cryst. Growth Des., Just Accepted Manuscript • DOI: 10.1021/acs.cgd.6b01658 • Publication Date (Web): 20 Feb 2017

Downloaded from http://pubs.acs.org on February 26, 2017

\section{Just Accepted}

"Just Accepted" manuscripts have been peer-reviewed and accepted for publication. They are posted online prior to technical editing, formatting for publication and author proofing. The American Chemical Society provides "Just Accepted" as a free service to the research community to expedite the dissemination of scientific material as soon as possible after acceptance. "Just Accepted" manuscripts appear in full in PDF format accompanied by an HTML abstract. "Just Accepted" manuscripts have been fully peer reviewed, but should not be considered the official version of record. They are accessible to all readers and citable by the Digital Object Identifier (DOI®). "Just Accepted" is an optional service offered to authors. Therefore, the "Just Accepted" Web site may not include all articles that will be published in the journal. After a manuscript is technically edited and formatted, it will be removed from the "Just Accepted" Web site and published as an ASAP article. Note that technical editing may introduce minor changes to the manuscript text and/or graphics which could affect content, and all legal disclaimers and ethical guidelines that apply to the journal pertain. ACS cannot be held responsible for errors or consequences arising from the use of information contained in these "Just Accepted" manuscripts. 
Cover Page:

\section{Microwave-assisted rapid synthesis of $\gamma$-cyclodextrin metal-organic}

3 frameworks for size control and efficient drug loading

4

5

6

$7 \mathrm{Guo}^{1}$, Fanyue Meng ${ }^{1}, \mathrm{Xu} \mathrm{Xu^{2 } , \text { Peter York }}{ }^{1}$, Zhaoxin Liu ${ }^{2,{ }^{*}}$, Jiwen Zhang ${ }^{1,2,3,{ }^{*}}$

8 Affiliations:

$9 \quad{ }^{1}$ Center for Drug Delivery Systems, Shanghai Institute of Materia Medica, Chinese Academy of 10 Sciences, Shanghai 201210, China

11

${ }^{2}$ School of Chemical and Environmental Engineering, Shanghai Institute of Technology, Shanghai 201418, China

${ }^{3}$ University of Chinese Academy of Sciences, Beijing 100049, China

${ }^{\text {a: }}$ Who contributed equally to the manuscript

\section{*Corresponding Author:}

Prof. Jiwen Zhang

Center for Drug Delivery Systems, Shanghai Institute of Materia Medica, Chinese Academy of Sciences, No. 501 of Haike Road, Shanghai 201210, China; Tel: +86-21-20231980; E-mail: jwzhang@simm.ac.cn.

Mr. Zhaoxin Liu

Shanghai Institute of Technology, No. 100, Haiquan Road, Shanghai 201418, China; Tel: +86-2160877215; E-mail: ericzxliu@sina.com. 


\section{Abstract}

30

The micron and nanometer sized $\gamma$-cyclodextrin metal-organic frameworks $(\gamma$-CD-MOFs) were successfully synthesized using microwave technique for the first time for rapid and facile synthesis. Polyethylene glycol 20000 (PEG 20000) was used as surfactant to control the size and morphology of $\gamma$-CD-MOFs. The as-synthesized $\gamma$-CD-MOFs were characterized using various techniques, including X-ray powder diffraction (PXRD), scanning electron microscopy (SEM), thermogravimetric analysis (TGA) and $\mathrm{N}_{2}$ adsorption. The increment in the reaction time and $\mathrm{MeOH}$ ratio dramatically damaged the crystalline integrity of $\gamma$-CD-MOFs. Fenbufen was selected as a model drug to evaluate the loading characteristics of $\gamma$-CD-MOF crystals. In results, the nanometer sized $\gamma$-CD-MOFs $(100-300 \mathrm{~nm})$ showed rapid and higher adsorption $\left(196 \mathrm{mg} \cdot \mathrm{g}^{-1}\right)$ of Fenbufen in EtOH when compared with the micron crystals. The adsorption parameters fitted well to a pseudo-second-order kinetic model and chemisorption of Fenbufen was further supported by molecular docking illustrations. In summary, the control synthesis of $\gamma$-CD-MOFs was successfully achieved by microwave assisted method and resultant crystals were further evaluated for potential drug delivery applications.

Keywords: Cyclodextrin-metal-organic frameworks; microwave; PEG 20000; drug loading; crystallinity 
49

50

51

52

53

54

55

56

57

58

59

60

61

62

63

64

65

66

67

68

69

70

71

72

\section{Title Page}

\section{Microwave-assisted rapid synthesis of $\gamma$-cyclodextrin metal-organic}

frameworks for size control and efficient drug loading

\section{Authors:}

Botao Liu ${ }^{1,2, a}$, Yaping $\mathrm{He}^{1,3, \mathrm{a}}$, Liping $\operatorname{Han}^{1}$, Vikramjeet $\operatorname{Singh}^{1}$, Xiaonan $\mathrm{Xu}^{1}$, Tao

Guo $^{1}$, Fanyue Meng ${ }^{1}, \mathrm{Xu} \mathrm{Xu}^{2}$, Peter York ${ }^{1}$, Zhaoxin $\mathrm{Liu}^{2, *}$, Jiwen Zhang ${ }^{1,2,3, *}$

Affiliations:

${ }^{1}$ Center for Drug Delivery Systems, Shanghai Institute of Materia Medica, Chinese Academy of Sciences, Shanghai 201210, China

${ }^{2}$ School of Chemical and Environmental Engineering, Shanghai Institute of Technology, Shanghai 201418, China

${ }^{3}$ University of Chinese Academy of Sciences, Beijing 100049, China

${ }^{\text {a: }}$ Who contributed equally to the manuscript

\section{Introduction}

Metal-organic frameworks (MOFs) have emerged as a new class of nanoporous materials with wide range of applications in molecular recognition, ${ }^{1}$ gas storage, ${ }^{2}$ catalysis ${ }^{3}$ and drug delivery. ${ }^{4}$ Usually, they are constructed from metal ion connectors and organic bridging ligands. ${ }^{5}$ Contrary to conventional porous material,,${ }^{6,7}$ the pore size and inner surface characteristics of MOFs can be modulated by tuning the size and shapes of the linkers. ${ }^{8}$

The sizes and shapes of MOF materials are critical for their various applications. Therefore, much efforts have been directed to shorten the synthesis time and to 
73 produce uniform crystals using microwave-assisted, ${ }^{6}$ mechanochemical $^{7}$ and 74 sonochemical ${ }^{8}$ methods. At the same time, several strategies have been adopted for 75 controlling the size and morphology of MOFs by altering the synthetic parameters 76 including temperature, processing duration, metal source and solvents. For example, 77 Ban et al reported the morphology control synthesis of ZIF-78 materials by adjusting 78 the nutrient and ligand concentrations. ${ }^{9}$ Pan et al reported a facile synthesis method 79 using cetyl trimethyl ammonium bromide as a capping agent for controlling the size 80 and morphology of ZIF-8 crystals in aqueous systems. ${ }^{10}$ Cheng et al presented a 81 solvothermal method for control synthesis of $\mathrm{NH}_{2}$-MIL-53 by altering the DMF and 82 water ratio without adding any surfactants or capping agents. ${ }^{11}$

83 In recent years, there has been a growing interest in encapsulating drugs in MOFs 84 (Table S1). However, it is very necessary to consider the biocompatibility of material 85 compositions for biomedical applications. Thus, appropriate natural molecules such as 86 amino acid, ${ }^{12}$ peptides ${ }^{13}$ and nucleobases ${ }^{14}$ as well as metal ions (Ca, Mg, Zn, Fe) are 87 considered to be biocompatible as organic linkers and metal connectors of MOFs, 88 respectively. In addition, some post-synthetic modifications of MOFs with biofriendly 89 functionalized linkers also showed their advantages over other reactive groups in $90 \quad$ various structures. ${ }^{15,16}$

91 Recently, Stoddart et al reported the synthesis of environmental friendly and 92 renewable cyclodextrin metal-organic frameworks (CD-MOFs) through a 93 vapor-diffusion method. ${ }^{17}$ The CD-MOFs are body-centered cubic extended structures 94 prepared from the coordination of $\gamma-\mathrm{CD}$ and potassium ion and possessed large 
95 spherical voids of $17 \AA$ with apertures of $7.8 \AA$. Among the various MOFs reported so 96 far, CD-MOFs are materials with potential to adsorb gases $\left(\mathrm{N}_{2}, \mathrm{H}_{2}, \mathrm{CO}_{2}\right.$ and $\left.\mathrm{CH}_{4}\right)$ and 97 some other molecules (Rhodamine B and 4-Phenylazophenol) within their pores. ${ }^{17}$ Taking advantage of their uniform channels (17 $\AA)$ and high local concentrations of $\mathrm{OH}^{-}$ions, the $\gamma$-CD-MOFs were used as template for the synthesis of silver and gold nanoparticles. $^{18}$

The original vapor diffusion method was able to produce cubic crystals $(40-500 \mu \mathrm{m})$

102 of $\gamma$-CD-MOFs at ambient temperature over the period of a week. ${ }^{17} \mathrm{~A}$ modified

103 method with the addition of CTAB and a controlled incubation time of 26-32 $\mathrm{h}$ has

104 been reported to produce $\gamma$-CD-MOF crystals, and they succeeded in the preparation

105 of good quality crystals with well-defined shape in the range of several hundred

106 nanometers to millimeters. ${ }^{19}$ However, vapor diffusion method is very difficult to

107 fabricate MOFs for mass production and future industrial use. Not long before, a

108 further improved approach for size control of $\gamma$-CD-MOFs has also been reported by

109 us with a conventional vapor diffusion technique, which took about 6 hours. ${ }^{20}$ In

110 addition, the previous size modulator of CTAB was quite toxic for cells.

111 In this paper, we report a fast synthesis of $\gamma$-CD-MOFs within several minutes under 112 microwave irradiation. More importantly, PEG 20000, a pharmaceutical excipient, 113 was used as the size modulator for the first time herein. In addition, we could 114 efficiently control the size and morphology of the obtained $\gamma$-CD-MOF crystals well 115 by optimizing the reaction time, temperature and solvent ratio in the synthesis process. 116 Fenbufen was selected as drug candidate to investigate the drug loading behavior of 
117 the crystals.

118 Experimental Section

119 Materials and Physical Measurements

$120 \gamma$-cyclodextrin $(\gamma$-CD, MaxDragon biochem Ltd), potassium hydroxide $(\mathrm{KOH}, 85.0 \%$,

121 Sinopharm Chemical Reagent Co., Ltd), methanol (MeOH, 99.5\%, Sinopharm

122 Chemical Reagent Co., Ltd), polyethylene glycol 20000 (PEG 20000, MW 20000,

123 Ourchem, Sinopharm Chemical Reagent Co., Ltd), ethanol (EtOH, 99.7\%, Sinopharm

124 Chemical Reagent Co., Ltd) and dichloromethane (DCM, 99.5\%, Sinopharm

125 Chemical Reagent Co., Ltd). Fenbufen (FBF, $>99.5 \%$ purity) was purchased from

126 Dalian Meilun Biotech Co., Ltd (China). Pure water (18.4 $\mathrm{M} \Omega \mathrm{cm})$ used in all

127 experiments was purified by a Milli-Q system (Millipore, Milford, MA, USA). All

128 other chemicals were of analytical grade and used without further purification.

129 Synthesis of $\boldsymbol{\gamma}$-CD-MOFs

130 A mother solution (Figure S1) was prepared by mixing $\gamma$-CD (324 mg) and KOH (112

$131 \mathrm{mg})$ in pure water $(10 \mathrm{~mL})$ with pre-addition of $6 \mathrm{~mL} \mathrm{MeOH}$, which was sealed and

132 placed in a glass vessel. The mixed solution was heated at $40 \sim 100{ }^{\circ} \mathrm{C}$ through

133 microwave irradiation (CEM, Discover, USA) with power $(100 \mathrm{w})$ for $1 \sim 120 \mathrm{~min}$

134 and the clear solution was obtained. Then $256 \mathrm{mg}$ of PEG 20000 was added quickly to

135 trigger the rapid deposition of crystalline materials (precipitation). 60 min later, the

136 micron sized MOF crystals were collected after separation, washed with $15 \mathrm{~mL}$ EtOH

137 and $\mathrm{MeOH}$ twice and dried overnight at $50^{\circ} \mathrm{C}$ under vacuum. In parallel experiments,

138 the size of the $\gamma$-CD-MOF crystals was modulated by altering the different processing 
139 parameters such as, reaction time $(t)$, temperature $(T)$, solvent ratio $(R)$ of water to

$140 \mathrm{MeOH}(\mathrm{v} / \mathrm{v})$ and modulators $(\mathrm{M})$. The synthesis procedure of nanometer sized crystals

141 was the same as that for micron sized $\gamma$-CD-MOFs. During the size modulation

142 process, $16 \mathrm{~mL}$ of $\mathrm{MeOH}$ with/without $128 \mathrm{mg}$ of PEG 20000 was added to the

143 reaction solution (F14 and F15) and the final solution was then heated at $50{ }^{\circ} \mathrm{C}$ for 10

144 min. The resulting samples were identified as F1 to F15, the conditions employed in

145 the controlled preparation and the morphology results of these samples are

146 summarized in Table 1. In comparison, the preparation of $\gamma$-CD-MOFs (identified as

147 F16) by conventional vapor diffusion method was also investigated according to

148 Smaldone's work (Supporting information S1). ${ }^{17}$

149

150 Table 1. Summary of synthesis conditions of F1-F15 samples

\begin{tabular}{|c|c|c|c|c|c|c|}
\hline Samples & $\begin{array}{c}\text { Heating time, } \\
t \text { (min) }\end{array}$ & $\begin{array}{c}\mathrm{T} \\
\left({ }^{\circ} \mathrm{C}\right)\end{array}$ & $\mathrm{R}$ & M & $\begin{array}{c}\text { Incubation time, } \\
t \text { (min) }\end{array}$ & Results (Morphology) \\
\hline F1 & 1 & 50 & 10: 6 & PEG 20000 & 60 & Typical cubes \\
\hline F2 & 10 & 50 & $10: 6$ & PEG 20000 & 60 & Typical cubes \\
\hline F3 & 20 & 50 & 10: 6 & PEG 20000 & 60 & Typical cubes \\
\hline F4 & 60 & 50 & 10: 6 & PEG 20000 & 60 & Typical cubes \\
\hline F5 & 120 & 50 & $10: 6$ & PEG 20000 & 60 & Typical cubes \\
\hline F6 & 10 & 40 & 10: 6 & PEG 20000 & 60 & Non-typical Cubes \\
\hline F7 & 10 & 60 & 10: 6 & PEG 20000 & 60 & Typical cubes \\
\hline F8 & 10 & 80 & 10: 6 & PEG 20000 & 60 & Typical cubes \\
\hline F9 & 10 & 100 & 10: 6 & PEG 20000 & 60 & Typical cubes \\
\hline F10 & 10 & 50 & $10: 4$ & PEG 20000 & 60 & Non-typical hexagonal shapes \\
\hline F11 & 10 & 50 & $10: 5$ & PEG 20000 & 60 & Non-typical Cubes \\
\hline F12 & 10 & 50 & 10: 7 & PEG 20000 & 60 & Typical cubes \\
\hline F13 & 10 & 50 & 10: 8 & PEG 20000 & 60 & Typical cubes \\
\hline F14 & 10 & 50 & 10: 6 & $\mathrm{MeOH}$ & 60 & Non-typical Cubes \\
\hline F15 & 10 & 50 & 10: 6 & $\mathrm{MeOH}+\mathrm{PEG} 20000$ & 60 & Non-typical Cubes \\
\hline
\end{tabular}


152

153

154

155

156

15

158

159

160

161

162

163

164

165

166

167

168

169

170

171

172

173

\section{Characterizations of $\gamma$-CD-MOFs}

Morphological characterizations of $\gamma$-CD-MOF crystals were conducted by the scanning electron microscope (SEM, S3400, Hitachi). The specimens were immobilized on a metal stub with double-sided adhesive tape and coated with a thin gold film, and then observed under definite magnification.

The crystallinity of the samples was characterized by X-ray powder diffraction (PXRD) analysis. Diffraction patterns of the prepared $\gamma$-CD-MOF crystals were detected with a Bruker D8 Advance diffractometer (Bruker, Germany) at ambient temperature, with tube voltage of $40 \mathrm{kV}$, tube current of $40 \mathrm{~mA}$ in a stepwise scan mode $\left(8^{\circ} \cdot \min ^{-1}\right)$. All the samples were irradiated with monochromatized CuKa radiation and analyzed over a $2 \theta$ angle range of $3-40^{\circ}$.

Thermogravimetric analysis (TGA) of $\gamma$-CD-MOF crystals was performed using a thermal analysis system (NETZSCH 209F3 240-20-382-L, USA) at a heating rate of $10{ }^{\circ} \mathrm{C} \cdot \mathrm{min}^{-1}$ under nitrogen. Samples were weighed (approx. $5 \mathrm{mg}$ ) in a hanging aluminum pan and the weight loss percentage of the samples was monitored from 30 to $400{ }^{\circ} \mathrm{C}$.

Nitrogen adsorption-desorption isotherm was measured with a liquid nitrogen bath $\left(-196{ }^{\circ} \mathrm{C}\right)$ using a porosimeter (Micromeritics ASAP 2020, USA). In order to remove the interstitial solvents, the samples were activated by immersing in dichloromethane for three days and dried under vacuum at $50{ }^{\circ} \mathrm{C}$ for $12 \mathrm{~h}$. Known amounts of samples (e. g. 150-200 mg) were loaded into the BET (Langmuir) sample tubes and degassed under vacuum $\left(10^{-5}\right.$ Torr) at $50{ }^{\circ} \mathrm{C}$ for $6 \mathrm{~h}$. BET (Langmuir) model was applied to 
174 measure the specific surface areas of the prepared samples.

175 FT-IR spectra of samples were obtained using an FT-IR spectrometer (Nicolet

176 Continuum XL, Thermo Fisher Scientific). Briefly, the sample and potassium bromide

177 were mixed well with a ratio of 1:10 followed by being compressed into a disk. 32

178 scans were carried out in wavenumber $400-4000 \mathrm{~cm}^{-1}$ at a resolution of $4 \mathrm{~cm}^{-1}$.

179 Adsorption experiment

180 In order to investigate the adsorption behavior of $\gamma$-CD-MOFs for FBF in EtOH 181 solution, $50 \mathrm{mg}$ of $\gamma$-CD-MOFs were added into $25 \mathrm{~mL}$ of FBF solution (600 $\left.182 \mu \mathrm{g} \cdot \mathrm{mL}^{-1}\right)$ at $30{ }^{\circ} \mathrm{C}$ temperature. The suspensions were shaken $(150 \mathrm{rpm})$ and 183 incubated for $24 \mathrm{~h}$. The FBF content of the solution was determined followed by a 184 HPLC method. The adsorption capacity (q) of $\gamma$-CD-MOFs towards FBF was 185 calculated as follows:

$186 \quad \mathrm{q}_{\mathrm{t}}=\frac{\mathrm{v}\left(\mathrm{C}_{0}-\mathrm{C}_{\mathrm{t}}\right)}{\mathrm{W}}$

187 where $\mathrm{q}_{\mathrm{t}}\left(\mu \mathrm{g} \cdot \mathrm{mg}^{-1}\right)$ is the adsorption capacity at contact time $\mathrm{t}, \mathrm{V}$ is the volume of 188 FBF solution $(\mathrm{mL}), \mathrm{C}_{0}$ is the initial concentration of FBF $\left(\mu \mathrm{g} \cdot \mathrm{mL}^{-1}\right), \mathrm{C}_{\mathrm{t}}$ is the 189 concentration of FBF at contact time $\mathrm{t}\left(\mu \mathrm{g} \cdot \mathrm{mL}^{-1}\right)$, and $\mathrm{W}$ is the weight of CD-MOFs 190 (mg).

191 Release of FBF from FBF loaded CD-MOFs in EtOH was also performed. And the 192 detailed methods and results were described in Supporting Information (S5 and Figure 193 S7).

\section{HPLC method for determination of FBF}

195 The analysis was carried out with an Agilent C18 column $(4.6 \mathrm{~mm} \times 150 \mathrm{~mm}, 3.6 \mu \mathrm{m}$ 196 i.d.) using flow rate of $1.0 \mathrm{~mL} \cdot \mathrm{min}^{-1}$ at a wavelength of $281 \mathrm{~nm}$. The FBF was 
197 detected with the column temperature of $25^{\circ} \mathrm{C}$, the injection volume of $2 \mu \mathrm{L}$ and the 198 mobile phase composed of $10 \%$ acetonitrile in $0.1 \%$ formic acid aqueous solution, 199 changing linearly over $10 \mathrm{~min}$ to $90 \%$ acetonitrile maintained for $3 \mathrm{~min}$, and then 200 decreasing to $10 \%$ in 1 min maintained for $6 \mathrm{~min}$.

\section{Molecular docking of FBF and $\gamma$-CD-MOFs}

202 The crystal structure of CD-MOFs was extracted from single crystal structure of 203 CD-MOFs in literature. ${ }^{21}$ In the docking model, an expanded non-periodic structure 204 was used, in which the $\mathrm{K}^{+}$ion that not affecting rigid docking results was deleted and 205 the $\mathrm{OH}^{-}$ion was replaced by $\mathrm{H}_{2} \mathrm{O}$. The structure of sucralose molecule was built using 206 the Materials Visualizer module in Materials Studio (MS, Accelrys Inc.) 5.0. The 207 Forcite module in MS was employed for minimization and molecular dynamics (MD) 208 simulation. The docking program AutoDock Vina 1.1.2 was used to perform the 209 automated molecular docking calculation. ${ }^{22}$ Detailed method was described in S4.

\section{Results and discussion}

211 In this study, $\gamma$-CD-MOFs were synthesized by a microwave irradiation method of $212 \gamma-\mathrm{CD}$ and $\mathrm{KOH}$ in a 1: 8 molar ratio under different reaction conditions. Cubic $213 \gamma$-CD-MOF crystals were obtained by raising the reaction temperature and 214 pre-addition of sufficient reaction solvent. To the best of our knowledge, this is the 215 first report on synthesis of $\gamma$-CD-MOFs using microwave irradiation method and PEG 216 as an efficient size modulator. The synthesis procedure was thoroughly optimized as 217 explained in following sections.

218 Effects of reaction parameters on crystal assembling

219 Initial investigations revealed that reaction time and solvent ratio were critical to the 220 fabrication of $\gamma$-CD-MOFs crystals in microwave irradiation method. Different time 
parameters from $1 \mathrm{~min}$ to $120 \mathrm{~min}$ were considered for the optimization of reaction

222 time. The SEM images of the crystals synthesized at different time intervals revealed 223 the uniform cubic morphologies as shown in Figure 1a. The size of $\gamma$-CD-MOFs crystals $(1-3 \mu \mathrm{m})$ just modulated by PEG 20000 were recorded smaller when compared with those obtained with CTAB by vapor diffusion method, ${ }^{19}$ which might be due to the higher number of nucleation sites.

The PXRD results in Figure 1b suggested the high crystallinity of the samples synthesized at different time intervals in agreement with the crystals synthesized by conventional method (Figure S2) and the reported literature. ${ }^{19}$ However, the dramatic

$\mathbf{a}$

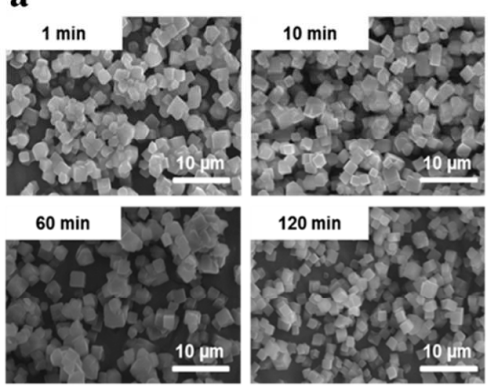

loss in crystallinity was recorded for prolonged reaction time of $120 \mathrm{~min}$ in spite of their cubic shapes. The microwave thermal effects are characterized as a local heating state. While the heating time is increased beyond the optimum level, such deterioration in cubic structure of CD-MOFs may be observed to some extent. Similar phenomenon was also found in the synthetic process of some other samples. ${ }^{23}$

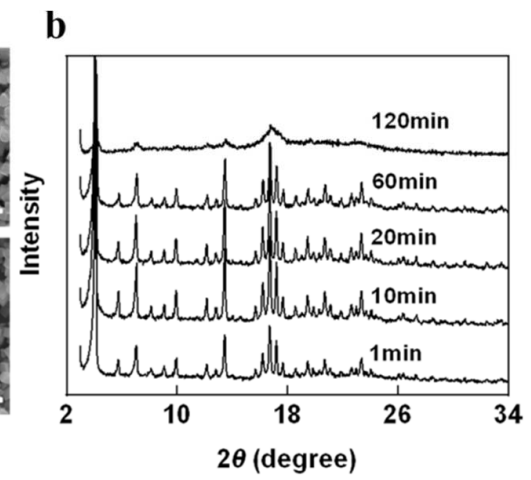

Figure 1. SEM morphology images and PXRD crystallinity patterns of $\gamma$-CD-MOF crystals obtained after different time of 1 (F1), 10 (F2), 60 (F4) and 120 (F5) min. The longer reaction time of 120 min showed the destruction of the crystalline structure of $\gamma$-CD-MOFs. 
241 In addition to the reaction time, the effect of temperature on the size and morphology

242 of $\gamma$-CD-MOFs was also investigated. SEM images of $\gamma$-CD-MOF crystals obtained at 243 different reaction temperature of $40,60,80$ and $100{ }^{\circ} \mathrm{C}$ at 10 min were shown in 244 Figure 2a. A significant effect of reaction temperature on the size of $\gamma$-CD-MOF 245 crystals was recorded. At lower temperatures, the deposition of white precipitates 246 were observed with the pre-addition of $\mathrm{MeOH}$ into the $\gamma-\mathrm{CD} / \mathrm{KOH}$ mother solution. 247 The precipitates did not dissolve completely at $40{ }^{\circ} \mathrm{C}$ and this observation can be 248 attributed to the rapid over-saturation of the precursors due to pre-addition of 249 excessive $\mathrm{MeOH}$. During the crystallization process, the anti-solvent recrystallization 250 process would be easier and the size of the newly obtained crystals would be 251 functioned by the recrystallization and the size modulator of PEG 20000, finally led to 252 the formation of smaller size of $\gamma$-CD-MOF crystals. In order to better control crystals 253 size, the increase of the temperature must be processed. With an further increase of 254 temperature from $50{ }^{\circ} \mathrm{C}$ to $100{ }^{\circ} \mathrm{C}$, no distinct influence on the size and morphology 255 of $\gamma$-CD-MOFs crystals was observed. The crystalline structure (Figure $2 \mathrm{~b}$ ) of $256 \gamma$-CD-MOFs does not change with the reaction temperature.
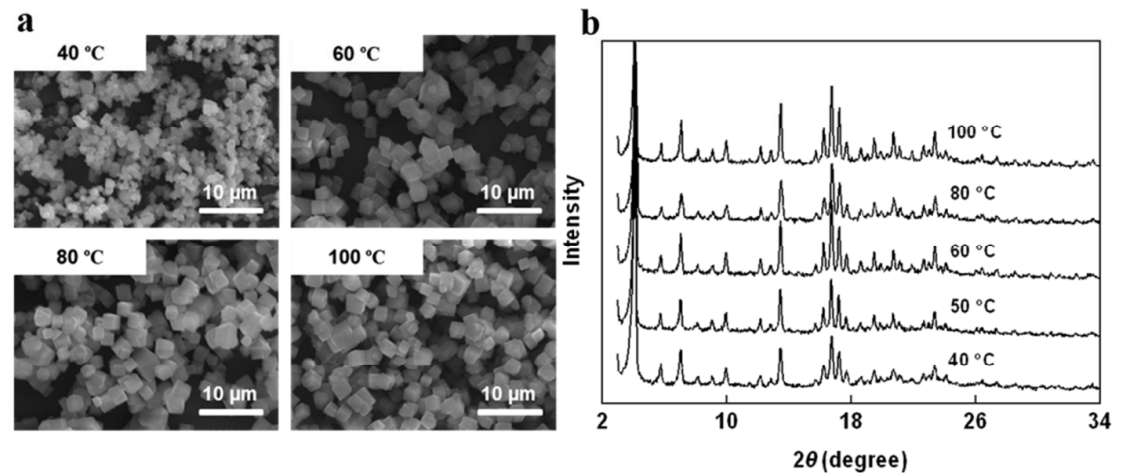

257

258

259

260

261

262

Figure 2. SEM morphology images and PXRD crystallinity patterns of $\gamma$-CD-MOF crystals obtained at different temperature of 40 (F6), 60 (F7), 80 (F8) and $100{ }^{\circ} \mathrm{C}$ (F9). The increase of temperature from 40 to $100{ }^{\circ} \mathrm{C}$ showed no influence on the crystalline structure of $\gamma$-CD-MOFs.

$\gamma$-CD-MOF crystals with different morphologies were obtained by varying the solvent 
ratio $\left(\mathrm{MeOH}\right.$ in $\left.\mathrm{MeOH}-\mathrm{H}_{2} \mathrm{O}\right)$ at $10 \mathrm{~min}$ and $50{ }^{\circ} \mathrm{C}$. Figure 3a showed the SEM images

264 of the samples synthesized with different water to $\mathrm{MeOH}$ ratios. Initially with low

$\mathrm{MeOH}$ volume, the irregular hexagonal crystals were produced. With increasing the proportion of $\mathrm{MeOH}$ to $37.5 \mathrm{vol} \%$ at same water content, the uniform cubic crystals were obtained. It was speculated that increment in $\mathrm{MeOH}$ volume contributes to the

277

278

279

280

281

282

283 disturbance of the crystallinity of $\gamma$-CD-MOFs.

\section{Size modulator effects} nucleation of MOF crystals due to the thermodynamic stability of crystal face growth. Crystal shape is often a consequence of the coexistence of slower and faster growth facets. With the growth of the crystal, the crystal morphology is dominated by the slower growth facets. ${ }^{24}$ Crystalline patterns of $\gamma$-CD-MOFs synthesized with different solvent ratios were presented in Figure $3 \mathrm{~b}$. It is well-known that the low supersaturation often leads to a decrease in nucleation sites. ${ }^{25}$ We expected that less volume of $\mathrm{MeOH}$ does not satisfy the level of supersaturation sufficient for the crystals growth. However, it was also observed that excessive volume of $\mathrm{MeOH}$ (water: $\mathrm{MeOH}=10: 8$ ) would also deteriorate the crystallinity of $\gamma$-CD-MOFs.

a
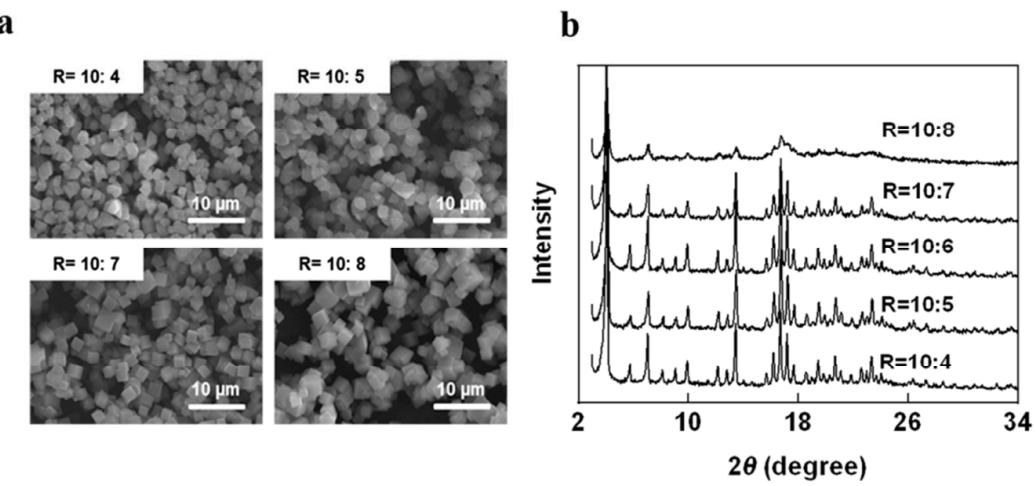

Figure 3. SEM morphology images and PXRD crystallinity patterns of $\gamma$-CD-MOF crystals obtained with different ratios of $\mathrm{H}_{2} \mathrm{O}$ to $\mathrm{MeOH}$ as 10: 4 (F10), 10: 5 (F11), 10: 7 (F12) and 10: 8 (F13). Higher volume ratios of $\mathrm{MeOH}$ resulted in $\gamma$-CD-MOFs more uniform but caused 
284 The smaller crystal size in nanometer range could be promising for biological 285 applications and traditional selective separation and catalysis. The crystal size can be 286 adjusted by controlling the nucleation and crystal growth rate. ${ }^{26}$ Micron sized $287 \gamma$-CD-MOF crystals can be obtained by simply adding the PEG 20000 as surfactant. $288 \mathrm{MeOH}$ was employed as a size modulator to obtain the nanometer sized crystals. 289 Crystal size of 200-800 nm was recorded by SEM as shown in Figure 4. Furthermore, 290 much smaller crystals of 100-300 nm were obtained by pre-mixing of $\mathrm{MeOH}$ with 291 PEG 20000 during modulation process. The crystallinity of nano crystals were found 292 consistent with those of micron sized crystals as shown in Figure 4. Smaller crystals 293 are usually obtained when the nucleation rate is larger than the rate of crystal 294 growth. ${ }^{27}$ It could be easily understood that excess volume of $\mathrm{MeOH}$ contributed to 295 the oversaturation of the reaction solution, finally resulted in a dramatic decrease of 296 CD-MOF size.
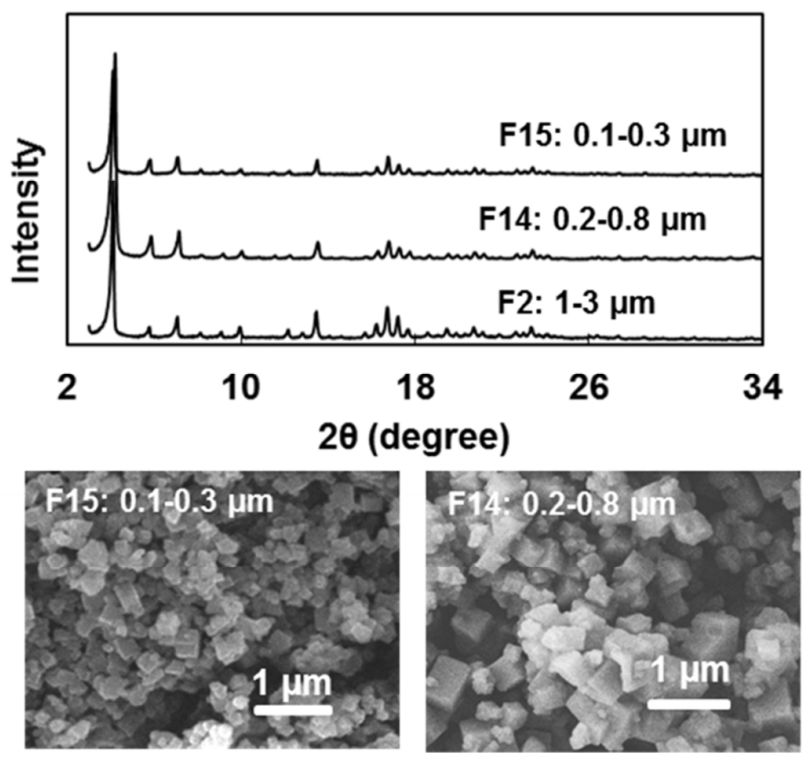

Figure 4. PXRD crystallinity patterns and SEM morphology images of $\gamma$-CD-MOF crystals. It 299 shows different sizes of $\gamma$-CD-MOFs possess the similar PXRD pattern. 
301 Adsorption isotherms of FBF on $\boldsymbol{\gamma}$-CD-MOFs

302 TGA data (Figure S 4) for DCM treated samples revealed a thermal stability region of 303 crystals following the initial loss due to residual solvent guest molecules. These 304 results directed us to evaluate the porosity of CD-MOF crystals. Not long before, a 305 total of 21 types of model drugs were screened to testify the adsorption capacity of $306 \gamma$-CD-MOFs, wherein, $\gamma$-CD-MOFs showed the highest captopril adsorption 307 capability which reached to $19.3 \%(\mathrm{w} / \mathrm{w}) .{ }^{20}$ Later, sucralose, a kind of non-nutritive 308 sweetener was loaded by $\gamma$-CD-MOFs and the thermal stability of this drug was 309 successfully improved, in which the drug loading efficiency for CD-MOF-Micro and CD-MOF-Nano was $17.5 \pm 0.9 \%$ and $27.9 \pm 1.4 \%(\mathrm{w} / \mathrm{w})$, respectively. ${ }^{28}$

311 The $\mathrm{N}_{2}$ adsorption-desorption isotherms (Figure 5) of activated $\gamma$-CD-MOFs of F15 $312(0.1-0.3 \mu \mathrm{m}), \mathrm{F} 14(0.2-0.8 \mu \mathrm{m}), \mathrm{F} 2(1-3 \mu \mathrm{m})$ and F16 $(40-500 \mu \mathrm{m})$ (Figure S3) 313 defined a BET (Langmuir) surface area of 673 (751), 1010 (1175), 820 (913) and $3141002(1118) \mathrm{m}^{2} \cdot \mathrm{g}^{-1}$, respectively. The above BET surface area results clearly 315 illustrated that the surface area of samples of F14 and F16 were larger than others, 316 which obviously indicated that the size modulator of PEG 20000 diminished the BET 317 surface area of $\gamma$-CD-MOF crystals. The sample of F15 possessed the lowest BET 318 surface area among these four samples, which could also be due to that some cavities 319 of CD-MOFs being blocked by PEG 20000 molecules. However, the drug adsorption 320 properties of $\gamma$-CD-MOFs crystals could not be directly estimated from BET results. 321 Thus, systematic adsorption experiments were set up to optimize their drug adsorption 322 abilities. 
323 Fenbufen, an analgesic and non-steroidal anti-inflammatory drug with a low aqueous

324 solubility and weak acidic nature, was selected for adsorption evaluations. The

325 dimensions of aperture window (7.8 $\AA)$ and internal pores $(17 \AA)$ are sufficiently large

326 to accommodate the FBF because of its small molecular size. In view of MOFs with

327 different sizes showing different adsorption capabilities towards same small molecular

328 sized compounds ${ }^{29}$, the adsorption capability to FBF was investigated using micron

329 and nanometer sized $\gamma$-CD-MOFs. The specific sizes and BET (Langmuir) surface

330 area results of used $\gamma$-CD-MOFs crystals are detailed in Table 2.
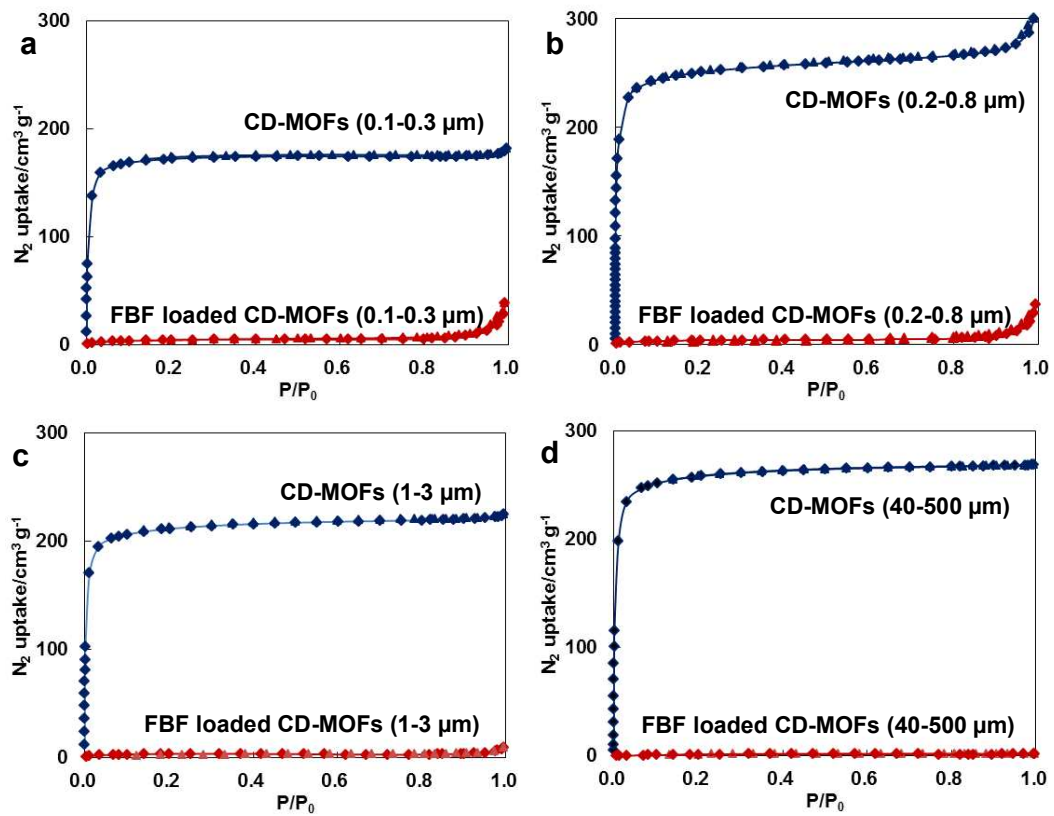

331

332

333

334

335

336

337

338

339

Figure 5. $\mathrm{N}_{2}$ Adsorption isotherms, for activated and FBF-loaded samples of $\gamma$-CD-MOFs of (a) F15 (0.1-0.3 $\mu \mathrm{m})$, (b) F14 (0.2-0.8 $\mu \mathrm{m})$, (c) F2 (1-3 $\mu \mathrm{m})$ and (d) F16 $(40-500 \mu \mathrm{m})$ measured at 77 $\mathrm{K}$. The $\mathrm{N}_{2}$ uptake defines a BET (Langmuir) surface area of 673 (751) (F15), 1010 (1175) (F14), $820(913)(\mathrm{F} 2)$ and $1002(1118)(\mathrm{F} 16) \mathrm{m}^{2} \cdot \mathrm{g}^{-1}$.

The effect of FBF incubation time on adsorption capacity is shown in Figure 6a. Gradual increment in adsorption content was noticed with the prolongation of time but varied in micron and nanometer sized $\gamma$-CD-MOFs. The crystals of 100-300 nm 
340 (F15) exhibited a rapid and higher adsorption capacity for FBF compared with other $341 \gamma$-CD-MOFs. The F15 sample showed a rapid adsorption during the first $1 \mathrm{~h}$ and 342 reached the adsorption equilibrium within $2 \mathrm{~h}$ with the highest adsorption capacity of $343196 \mathrm{mg} \cdot \mathrm{g}^{-1}$ (molar ratio of FBF to CD-MOFs was 1: 1.9). Obviously, the adsorption 344 content of sample F15 in 5 min was similar to that of sample F16 within $24 \mathrm{~h}$.

345 The obtained data was fitted well to pseudo-second-order kinetic model (see S3 for 346 method details) which suggested the chemisorption behavior of drug adsorption. ${ }^{30,31}$

347 The coefficients for the linear plots of $t / q_{t}$ against time for pseudo-second-order 348 kinetics were greater than 0.99 for all systems except $F 16\left(r^{2}=0.95\right.$, Figure $\left.6 \mathrm{~b}\right)$ which 349 might be due to the non-uniformity of F16 crystal sizes. The proposed hypothesis that 350 the FBF molecules occupied most of the crystals cavities was supported by a dramatic 351 decrease in the surface areas of the F2, F14, F15 and F16 crystals (Table 2 and Figure 352 5). Furthermore, the release rates of FBF loaded $\gamma$-CD-MOFs in EtOH were very 353 similar with the adsorption process and the cumulative release percentages of the four 354 samples within $20 \mathrm{~h}$ kept 70-85\% (Figure S7).

Table 2. Summary of the size of $\gamma$-CD-MOFs (F2, F14, F15, F16)

\begin{tabular}{ccc}
\hline Samples & Size $(\mu \mathrm{m})$ & BET $\left(\right.$ Langmuir) surface area $\left(\mathrm{m}^{2} \cdot \mathrm{g}^{-1}\right)$ \\
\hline F2 & $1-3$ & $820(913)$ \\
F14 & $0.2-0.8$ & $1010(1175)$ \\
F15 & $0.1-0.3$ & $673(751)$ \\
F16 & $40-500$ & $1002(1118)$ \\
\hline
\end{tabular}



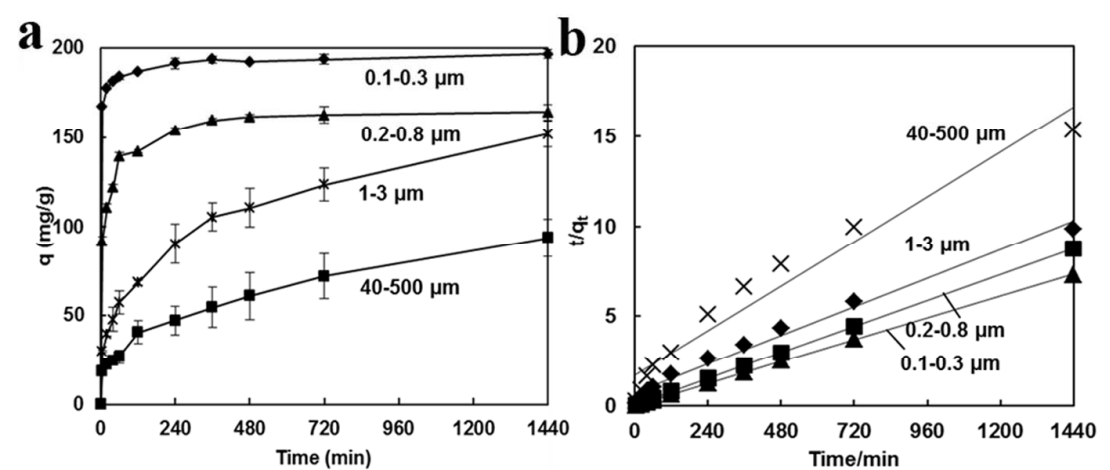

Figure 6. (a) Effects of contacted time on the adsorption of FBF onto Micro and Nanometer sized $\gamma$-CD-MOFs ( $\mathrm{n}=2)$ of F15 $(0.1-0.3 \mu \mathrm{m})$, F14 $(0.2-0.8 \mu \mathrm{m}), \mathrm{F} 2(1-3 \mu \mathrm{m})$ and F16 $(40-500 \mu \mathrm{m})$. The crystals with smaller size distinctly show a higher adsorption capacity than larger size CD-MOF crystals. (b) The fitting results of the pseudo-second-order kinetics fit the experimental data well.

\section{FT-IR spectra and molecular docking of FBF and $\gamma$-CD-MOFs}

The FT-IR spectra of FBF loaded $\gamma$-CD-MOFs (F2) samples are shown in Figure 7 in comparison with $\gamma$-CD-MOFs and pure FBF. The characteristic $\mathrm{C}=\mathrm{O}$ stretching vibrations at $1712 \mathrm{~cm}^{-1}$ (carboxylic acid) and $1679 \mathrm{~cm}^{-1}$ (ketone), the skeletal vibration of phenyl rings at $1600 \mathrm{~cm}^{-1}$, asymmetric and symmetric vibration of carboxylate groups at 1561 and $1402 \mathrm{~cm}^{-1}$ were observed for pure FBF. The $\mathrm{C}=\mathrm{O}$ stretching vibrations at $1712 \mathrm{~cm}^{-1}$ disappear/shift after adsorption, providing an indication that FBF molecules are loaded in the cavities of $\gamma$-CD-MOFs rather than adsorbed on the surface of the composites.

In order to explain the mechanism of FBF loading by $\gamma$-CD-MOFs, computer based molecular docking studies of FBF and $\gamma$-CD-MOFs were undertaken. In the case of 1:2 molar ratio for $\gamma-\mathrm{CD}$ and $\mathrm{FBF}$ in $\gamma$-CD-MOFs, the docking free energy was recorded $-7.0 \mathrm{kcal} \cdot \mathrm{mol}^{-1}$ and $-8.5 \mathrm{kcal} \cdot \mathrm{mol}^{-1}$ (Figure S5) for the first and second molecules of FBF, respectively. The simulation results suggested that the two FBF molecules would be favorably positioned in the cavities of D- $\gamma$-CDs (dual $\gamma$-CD units) of $\gamma$-CD-MOFs and the cavity of each $\gamma$-CD included one FBF molecule (detailed docking results are described in S4). Figure S6 illustrated that H-bonds can be readily 

and potassium ions in $\gamma$-CD-MOFs.

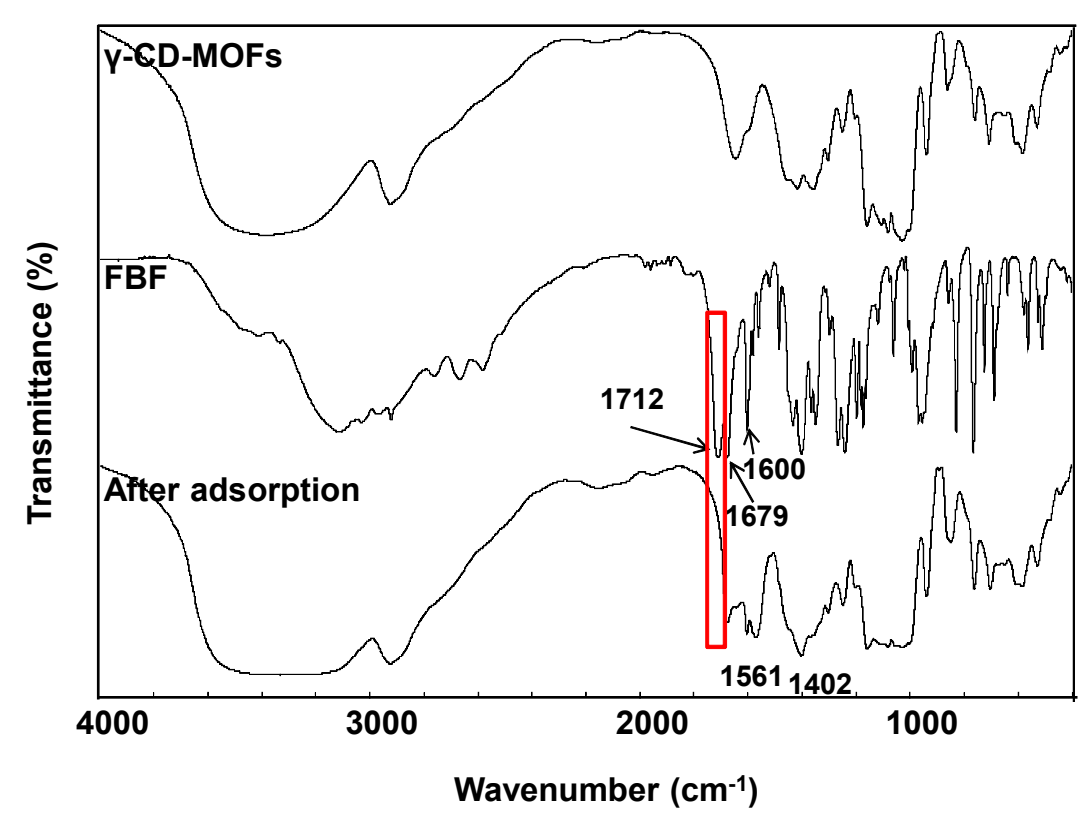

387

formed by the carbonyl (-COOH) of FBF with the hydroxyl of $\mathrm{D}-\gamma$-CDs in $\gamma$-CD-MOFs, supported by the shift of $\mathrm{C}=\mathrm{O}$ stretching vibrations at $1712 \mathrm{~cm}^{-1}$ to lower wave number in IR spectra. Considering the carboxyl function group and a small $\mathrm{pK}_{\mathrm{a}}$, the high adsorption capability of $\gamma$-CD-MOFs for FBF is believed to arise from the strong electrostatic interaction between the carbonyl group in FBF molecule

Figure 7. FT-IR spectra of $\gamma$-CD-MOFs, FBF and FBF loaded $\gamma$-CD-MOFs, respectively.

\section{Conclusions}

Microwave method for rapid and controlled synthesis of $\gamma$-CD-MOFs was reported. The developed method was able to shorten the hours' long fabrication process into minutes. The size and morphology of $\gamma$-CD-MOF crystals have been adjusted by altering the reaction time, temperature and solvent ratio. The PEG 20000 and/or $\mathrm{MeOH}$ were successfully employed as size modulators to obtain the nanometer sized crystals. Notably, an increase in the reaction time or $\mathrm{MeOH}$ ratio was found to 
397 damage the $\gamma$-CD-MOF crystallinity. The nanometer sized $\gamma$-CD-MOFs exhibited a

398 faster and higher adsorption capability of $196 \mathrm{mg} \cdot \mathrm{g}^{-1}$ for FBF within $24 \mathrm{~h}$ compared 399 with the micron sized. Adsorption kinetics of FBF towards $\gamma$-CD-MOFs $(600 \mu \mathrm{g}$. $400 \mathrm{~mL}^{-1}$ in EtOH) is described by the pseudo-second-order kinetic model. Molecular 401 docking further illustrated that FBF is likely to be chemisorbed by $\gamma$-CD-MOFs. Thus, 402 facile synthesis and size control approaches, together with FBF loading behavior of $403 \gamma$-CD-MOF crystals are providing support for their potential applications in drug 404 delivery.

405 Acknowledgements

406 We are grateful for the financial support from Natural Science Foundation of 407 China $(81373358,81430087)$ and National Science and Technology Major Project 408 (2013ZX09402103).

\section{Supporting Information}

410 Figure S1 to S7 showing synthesis scheme, SEM, PXRD, molecular 411 docking results and FBF release.

\section{References}

414

415

416

417

418

419

420

421

422

423

424

(1) Liao, W. M.; Shi, H. T.; Shi, X. H.; Yin, Y. G., Pyrolytic cavitation, selective adsorption and molecular recognition of a porous Eu(III) MOF. Dalton Transactions 2014, 43, (41), 15305-15307.

(2) Luebke, R.; Weseliński, Ł. J.; Belmabkhout, Y.; Chen, Z.; Wojtas, Ł.; Eddaoudi, M., Microporous Heptazine Functionalized (3,24)-Connectedrht-Metal-Organic Framework: Synthesis, Structure, and Gas Sorption Analysis. Crystal Growth \& Design 2014, 14, (2), 414-418.

(3) Nguyen, H. G. T.; Weston, M. H.; Sarjeant, A. A.; Gardner, D. M.; An, Z.; Carmieli, R.; Wasielewski, M. R.; Farha, O. K.; Hupp, J. T.; Nguyen, S. T., Design, Synthesis, Characterization, and Catalytic Properties of a Large-Pore Metal-Organic Framework Possessing Single-Site Vanadyl(monocatecholate) Moieties. Crystal Growth \& Design 2013, 13, (8), 3528-3534.

(4) Li, Q. L.; Wang, J. P.; Liu, W. C.; Zhuang, X. Y.; Liu, J. Q.; Fan, G. L.; Li, B. H.; Lin, W. N.; Man, J. H., A new $(4,8)$-connected topological MOF as potential drug delivery. Inorg. Chem. Commun. 2015, 55, 
8-10.

426 (5) Zou, G.-D.; He, Z.-Z.; Tian, C.-B.; Zhou, L.-J.; Feng, M.-L.; Zhang, X.-D.; Huang, X.-Y., Microwave and Conventional Hydro(solvo)thermal Syntheses of Three Co(II) Coordination Polymers: Supramolecular Isomerism and Structural Transformations Accompanied by Tunable Magnetic Properties. Crystal Growth \& Design 2014, 14, (9), 4430-4438.

430

(6) Khan, N. A.; Jhung, S. H., Synthesis of metal-organic frameworks (MOFs) with microwave or ultrasound: Rapid reaction, phase-selectivity, and size reduction. Coord. Chem. Rev. 2015, 285, 11-23.

432

433

434

435

436

437

438

439

440

441

442

443

444

445

446

447

448

449

450

451

452

453

454

455

456

457

458

459

460

461

462

463

464

465

466

467

468

(7) Pilloni, M.; Padella, F.; Ennas, G.; Lai, S.; Bellusci, M.; Rombi, E.; Sini, F.; Pentimalli, M.; Delitala, C.; Scano, A., Liquid-assisted mechanochemical synthesis of an iron carboxylate Metal Organic Framework and its evaluation in diesel fuel desulfurization. Microporous \& Mesoporous Materials 2015, 213, 14-21.

(8) Bigdeli, M.; Morsali, A., Sonochemical synthesis of a nano-structured zinc(II) amidic pillar metalorganic framework. Ultrason. Sonochem. 2015, 27, 416-422.

(9) Ban, Y.; Li, Y.; Liu, X.; Peng, Y.; Yang, W., Solvothermal synthesis of mixed-ligand metal-organic framework ZIF-78 with controllable size and morphology. Microporous Mesoporous Mater. 2013, 173, 29-36.

(10) Pan, Y.; Heryadi, D.; Zhou, F.; Zhao, L.; Lestari, G.; Su, H.; Lai, Z., Tuning the crystal morphology and size of zeolitic imidazolate framework-8 in aqueous solution by surfactants. CrystEngComm 2011, 13, (23), 6937.

(11) Cheng, X.; Zhang, A.; Hou, K.; Liu, M.; Wang, Y.; Song, C.; Zhang, G.; Guo, X., Size- and morphology-controlled NH2-MIL-53(Al) prepared in DMF-water mixed solvents. Dalton Trans 2013, 42, (37), 13698-705.

(12) Hou, J.-J.; Xu, X.; Jiang, N.; Wu, Y.-Q.; Zhang, X.-M., Selective adsorption in two porous triazolateoxalate-bridged antiferromagnetic metal-azolate frameworks obtained via in situ decarboxylation of 3-amino-1,2,4-triazole-5-carboxylic acid. J. Solid State Chem. 2015, 223, 73-78.

(13) Katsoulidis, A. P.; Park, K. S.; Antypov, D.; Marti-Gastaldo, C.; Miller, G. J.; Warren, J. E.; Robertson, C. M.; Blanc, F.; Darling, G. R.; Berry, N. G.; Purton, J. A.; Adams, D. J.; Rosseinsky, M. J., Guest-adaptable and water-stable peptide-based porous materials by imidazolate side chain control. Angew. Chem. Int. Ed. Engl. 2014, 53, (1), 193-8.

(14) Thomas-Gipson, J.; Pérez-Aguirre, R.; Beobide, G.; Castillo, O.; Luque, A.; Pérez-Yáñez, S.; Román, P., Unravelling the Growth of Supramolecular Metal-Organic Frameworks Based on Metal-Nucleobase Entities. Crystal Growth \& Design 2015, 15, (2), 975-983.

(15) Hintz, H.; Wuttke, S., Postsynthetic modification of an amino-tagged MOF using peptide coupling reagents: a comparative study. Chem. Commun. (Camb.) 2014, 50, (78), 11472-5.

(16) Bonnefoy, J.; Legrand, A.; Quadrelli, E. A.; Canivet, J.; Farrusseng, D., Enantiopure Peptide-Functionalized Metal-Organic Frameworks. J. Am. Chem. Soc. 2015, 137, (29), 9409-16.

(17) Smaldone, R. A.; Forgan, R. S.; Furukawa, H.; Gassensmith, J. J.; Slawin, A. M.; Yaghi, O. M.; Stoddart, J. F., Metal-organic frameworks from edible natural products. Angew. Chem. Int. Ed. Engl. 2010, 49, (46), 8630-4.

(18) Wei, Y.; Han, S.; Walker, D. A.; Fuller, P. E.; Grzybowski, B. A., Nanoparticle core/shell architectures within MOF crystals synthesized by reaction diffusion. Angew. Chem. Int. Ed. Engl. 2012, 51, (30), 7435-9.

(19) Furukawa, Y.; Ishiwata, T.; Sugikawa, K.; Kokado, K.; Sada, K., Nano- and Microsized Cubic Gel Particles from Cyclodextrin Metal-Organic Frameworks †. Angew. Chem. Int. Ed. 2012, 51, (42), 
10566-10569.

(20) Botao Liu, H. L., Xiaonan Xu, Xue Li, Nana Lv, Vikramjeet Singh, J. Fraser Stoddart, Peter York, Xu $\mathrm{Xu}$, Ruxandra Gref, Jiwen Zhang, Optimized synthesis and crystalline stability of $\gamma$-cyclodextrin metal-organic frameworks for drug adsorption. Int. J. Pharm. 2016.

473 (21) Forgan, R. S.; Smaldone, R. A.; Gassensmith, J. J.; Furukawa, H.; Cordes, D. B.; Li, Q.; Wilmer, C. E.; Botros, Y. Y.; Snurr, R. Q.; Slawin, A. M.; Stoddart, J. F., Nanoporous carbohydrate metal-organic frameworks. J. Am. Chem. Soc. 2012, 134, (1), 406-17.

(22) Trott, O.; Olson, A. J., AutoDock Vina: Improving the speed and accuracy of docking with a new scoring function, efficient optimization, and multithreading. J. Comput. Chem. 2010, 31, (2), 455-461. (23) Choi, J.-S.; Son, W.-J.; Kim, J.; Ahn, W.-S., Metal-organic framework MOF-5 prepared by microwave heating: Factors to be considered. Microporous Mesoporous Mater. 2008, 116, (1-3), 480 727-731.

481 (24) Umemura, A.; Diring, S.; Furukawa, S.; Uehara, H.; Tsuruoka, T.; Kitagawa, S., Morphology design 482 of porous coordination polymer crystals by coordination modulation. J. Am. Chem. Soc. 2011, 133, 483 (39), 15506-13.

484 (25) Brar, T.; Paul France, A.; Smirniotis, P. G., Control of Crystal Size and Distribution of Zeolite A. Ind. 485 Eng. Chem. Res. 2001, 40, (4).

486 (26) Lethbridge, Z. A. D.; Williams, J. J.; Walton, R. I.; Evans, K. E.; Smith, C. W., Methods for the 487 synthesis of large crystals of silicate zeolites. Microporous Mesoporous Mater. 2005, 79, (1-3), $488 \quad 339-352$.

489 (27) Jhung, S. H.; Ji, H. L.; Chang, J. S., Crystal size control of transition metal ion-incorporated 490 aluminophosphate molecular sieves: Effect of ramping rate in the syntheses. Microporous \& 491 Mesoporous Materials 2008, 112, (1-3), 178-186.

492 (28) Lv, N.; Guo, T.; Liu, B.; Wang, C.; Singh, V.; Xu, X.; Li, X.; Chen, D.; Gref, R.; Zhang, J., Improvement 493 in Thermal Stability of Sucralose by gamma-Cyclodextrin Metal-Organic Frameworks. Pharm. Res. 2016.

496

(29) Chen, M.; Wang, Z.; Han, D.; Gu, F.; Guo, G., High-sensitivity NO2 gas sensors based on flower-like 497 and tube-like ZnO nanomaterials. Sensors Actuators B: Chem. 2011, 157, (2), 565-574. 

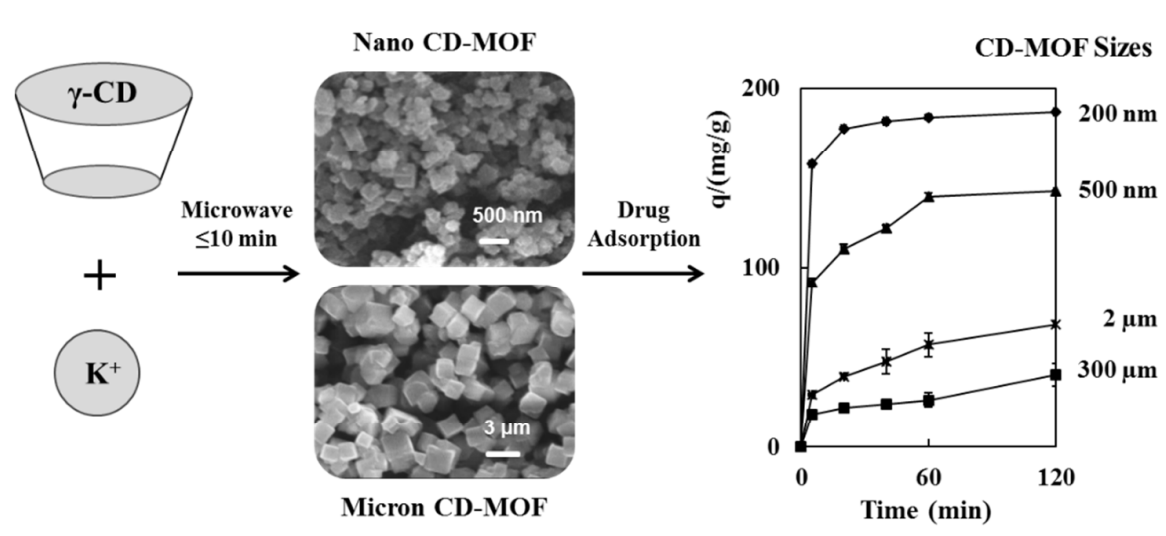

Manuscript title:

Microwave-assisted rapid synthesis of $\gamma$-cyclodextrin metal-organic frameworks for size control and efficient drug loading

\section{Author list:}

Botao Liu ${ }^{1,2, a}$, Yaping $\mathrm{He}^{1,3, \mathrm{a}}$, Liping $\mathrm{Han}^{1}$, Vikramjeet $\operatorname{Singh}^{1}$, Xiaonan $\mathrm{Xu}^{1}$, Tao Guo $^{1}$, Fanyue Meng ${ }^{1}, \mathrm{Xu} \mathrm{Xu}^{2}$, Peter York ${ }^{1}$, Zhaoxin Liu ${ }^{2, *}$, Jiwen Zhang ${ }^{1,2,3, *}$

\section{Affiliations:}

${ }^{1}$ Center for Drug Delivery Systems, Shanghai Institute of Materia Medica, Chinese Academy of Sciences, Shanghai 201210, China

${ }^{2}$ School of Chemical and Environmental Engineering, Shanghai Institute of Technology, Shanghai 201418, China

${ }^{3}$ University of Chinese Academy of Sciences, Beijing 100049, China

${ }^{\mathrm{a}}$ Who contributed equally to the manuscript

\section{TOC graphic:}

Synopsis:

$\gamma$-CD-MOFs were synthesized by microwave-assisted technique for the first time and exploited for drug delivery applications. The size and morphology of $\gamma$-CD-MOF crystals can be efficiently controlled by optimizing the synthesis process. Compared with micron crystals, nanometer sized $\gamma$-CD-MOFs $(100-300 \mathrm{~nm})$ showed rapid and higher adsorption (196 mg $\mathrm{g}^{-1}$ ) of Fenbufen which implies the good loading characteristics of $\gamma$-CD-MOFs. 